\title{
Double $K$-shell ionization of Al induced by photon and electron impact
}

\author{
K. Fennane, J.-Cl. Dousse, ${ }^{*}$ J. Hoszowska, M. Berset, W. Cao, Y.-P. Maillard, J. Szlachetko, ${ }^{\dagger}$ and M. Szlachetko \\ Department of Physics, University of Fribourg, CH-1700 Fribourg, Switzerland \\ M. Kavčič \\ J. Stefan Institute, SI-1001 Ljubljana, Slovenia
}

\begin{abstract}
This paper reports on the investigation of the double $K$-shell ionization of metallic aluminum induced by photon and electron impact. The experimental method consisted to measure the $K \alpha$ hypersatellite spectra resulting from the radiative decay of double $1 s$ vacancy states by means of high-resolution X-ray spectroscopy using a Bragg-type von Hamos crystal spectrometer. Measurements of the photon-induced spectra were carried out at the European Synchrotron Radiation Facility, Grenoble, France, while those of the electron-induced spectra were performed at the University of Fribourg. The double $1 s$ ionization probabilities and cross sections were derived from the $K \alpha_{2}$ hypersatellite to diagram line intensity ratios for photon beam energies ranging between 3.1 and $5.5 \mathrm{keV}$ and for different incident electron-beam energies ranging between 4 and $20 \mathrm{keV}$. The energy and linewidth of the $K \alpha_{2}$ hypersatellite $\mathrm{x}$-ray line were also determined for which consistent results were obtained from the photon and electron-beam measurements.
\end{abstract}

PACS number(s): 34.80.Dp, 32.30.Rj, 32.70. -n, 32.80.Fb

\section{INTRODUCTION}

Hollow $K$-shell atoms are atoms in which the innermost shell is empty while the outer shells are occupied. They can be created either by double $K$-shell ionization of neutral atoms or via multiple electron capture (EC) into outer shells by fully stripped ions in interaction with metallic surfaces. The radiative de-excitation of hollow $K$-shell atoms leads to the emission of so-called $K$ x-ray hypersatellites $\left(K \alpha^{h}, K \beta^{h}, \ldots\right)$. The intensity ratio of the $K \alpha_{1}^{h}\left(1 s^{-2} \rightarrow 1 s^{-1} 2 p_{3 / 2}^{-1}\right)$ to $K \alpha_{2}^{h}$ $\left(1 s^{-2} \rightarrow 1 s^{-1} 2 p_{1 / 2}^{-1}\right)$ hypersatellite lines is a very effective tool to study the variation in the coupling scheme with the atomic number $Z$. Precise measurements of the hypersatellite transition energies can also be used to learn more about the Breit interaction and relativistic effects. Because of the strong correlations between the two $1 s$ electrons, hollow atoms provide also a good opportunity to study the effects of electronelectron correlations. This aspect is particularly important since the concept of electron correlations is central in various areas of physics [1].

The double $K$-shell ionization can be produced via three main mechanisms, namely, the shake-off (SO), the knock-out (KO), and also the two-step-two (TS2) processes, in the case of charged particle impact. Shake off is a one-step mechanism in which one of the target electrons is removed by photoionization or by impact with a charged particle and then, because of the abrupt change in the atomic potential resulting from the first ionization, a second electron is ejected during the relaxation process of the remaining ion. The KO process is a two-step mechanism in which the first ionized electron hits a second bound electron which is then kicked out. This mechanism is alternatively referred to as a

\footnotetext{
*Corresponding author; jean-claude.dousse@unifr.ch

$\dagger$ On leave from Jan Kochanowski University, Institute of Physics, 25-406 Kielce, Poland.
}

two-step-one (TS1) process. The TS2 process is also a twostep mechanism; here, however, the two electrons are emitted by two subsequent interactions between the incident charged projectile and two electrons of the same target atom. In photoabsorption, the TS2 process is not possible since the incident photon is annihilated during the first interaction so that the double $K$-shell ionization can proceed only through electron-electron interactions. These interactions were long thought to be due mainly to ground-state correlations (GSCs) and SO processes. Using the many-body perturbation theory (MBPT), Carter and Kelly [2] and Ishihara et al. [3] demonstrated that a large contribution to the double photoabsorption probability originates from the KO process. This dynamical scattering picture was confirmed by Samson [4] who pointed out that experimental data of double to single photoionization cross sections of neutral atoms are proportional to the electron-impact ionization cross sections of singly charged ions. At high photon energies the probability of Compton scattering is no more negligible and it could be even higher than the photoabsorption probability. In this scattering process the incident photon is not annihilated but the probability that it can make two successive Compton scatterings at two electrons of the same atom is negligible [5] so that at high photon energies electron-electron correlations represent also the single mechanism by which double $K$-shell ionization can be produced. For atomic collisions with light charged particles, in addition to the SO and TS1 processes, double ionization can also occur via the TS2 channel. The latter is expected to dominate for projectile velocities $v$ (in a.u.) and charges $q$ such that $q / v>0.2$ [6]. For heavier charged projectiles multi-ionization of atoms can also result from charge-transfer processes in which one or more target electrons are captured into an empty shell of the projectile. Charge transfer becomes important when heavy bare ions are used as projectiles.

The first experimental studies of hollow atoms have been made using radioactive samples [7-9]. In this case, the double $K$-shell ionization is also due to correlation effects. 
Valuable data were extracted from these studies. However, the disadvantage of this excitation mode is that radioactive sources with long enough half-lives are needed. In addition only those elements for which natural radioactive isotopes are available can be studied. As a consequence, only a limited number of elements could be investigated. The ratio of double to single $K$-shell photoionization cross sections was first observed for He by Carlson in 1967 [10]. In the same year Byron and Joachain [11] published the first calculations of this ratio, and since then, numerous experimental and theoretical studies have been done. Synchrotron radiation sources, allowing the production of monochromatic highly collimated and extremely intense photon beams at any energy, have revolutionized the experimental research on multiple photoionization. The first $K \alpha$ hypersatellite spectrum produced by means of synchrotron radiation was observed in 1999 by Kanter et al. [12] for Mo. Few similar measurements were performed for $\mathrm{Ca}, \mathrm{Ti}$, and V [13], V, Cr, Mn, Fe, $\mathrm{Co}, \mathrm{Ni}, \mathrm{Cu}$, and $\mathrm{Zn}$ [14-16], $\mathrm{Ne}$ [17], and $\mathrm{Ag}$ [18]. These experiments as well as those performed using charged particle impact, in addition to $(h \nu, 2 e)$ and $(e, 3 e)$ kinematics experiments $[19,20]$, have provided further insights to multiionization mechanisms.

In the present work which is a continuation of these efforts, we report on measurements of the $\mathrm{Al} K \alpha$ hypersatellite spectra. The latter were obtained by bombarding metallic aluminum foils with monoenergetic beams of photons and electrons at different energies. Besides the precise determination of the energy and linewidth of the Al $K \alpha^{h}$ hypersatellite $\mathrm{x}$-ray line, the aim of the present work was also to determine the energy dependence of both photon- and electroninduced double $K$-shell ionization cross sections from threshold to the expected broad maximum and to probe the current theoretical models describing this dependence. A further objective of our study was to compare the photon- and electron-induced double $K$-shell ionization cross sections in order to better understand the mechanisms by which the double $1 s$ vacancy production is achieved in each case.

\section{EXPERIMENT AND DATA ANALYSIS}

\section{A. Measurements with synchrotron radiation}

\section{Experimental setup}

The experiment was performed at the $\mathrm{x}$-ray microscopy beamline ID21 at the European Synchrotron Radiation Facility (ESRF), in Grenoble, France. The x-ray beam provided by an undulator was monochromatized with a double $\mathrm{Ni} / \mathrm{B}_{4} \mathrm{C}$ multilayer. The number of photons impinging on the target was typically $1-2 \times 10^{12} / \mathrm{s}$. Rejection of upper harmonics was realized using a Si-based Ni coated mirror. The energy calibration of the monochromator was done by measuring with a photodiode the $K$ absorption edges of $\mathrm{V}, \mathrm{Ti}, \mathrm{Ca}$, and $\mathrm{S}$.

The x-ray spectra were observed with the high-resolution reflection-type von Hamos crystal spectrometer of Fribourg [21]. The latter was installed in the main experimental hutch of ID21, downstream to the scanning x-ray microscope (SXM) chamber to which it was connected through a $180 \mathrm{~cm}$ long evacuated pipe. The basic elements of the von Hamos spectrometer are an x-ray source defined by a rectangular slit placed in front of the target, a cylindrically bent crystal, and a position sensitive detector, located on the crystal axis of curvature. The target consisted of a $99.1 \%$ pure metallic aluminum foil. In order to minimize the self-absorption of the hypersatellite $\mathrm{x}$ rays in the sample, a target thickness of $1 \mu \mathrm{m}$ was chosen. The target was tilted to $30^{\circ}$ relative to the direction of the incident photon beam. A slit width of $0.2 \mathrm{~mm}$ was used as the best compromise between an acceptable energy resolution and high enough luminosity, two key parameters for the measurements of the $\mathrm{Al} K \alpha$ hypersatellites. The emitted fluorescence $\mathrm{x}$ rays were reflected in first order by a $10 \mathrm{~cm}$ high $\times 5 \mathrm{~cm}$ wide $\times 1 \mathrm{~mm}$ thick ammonium dihydrogen phosphate (ADP) (101) crystal $(2 d=10.642 \AA)$. For the detection of the diffracted $\mathrm{x}$ rays, a $26.8 \mathrm{~mm}$ long $\times 8 \mathrm{~mm}$ high back-illuminated charge coupled device (CCD) camera with a pixel size of $20 \times 20 \mu \mathrm{m}^{2}$ was employed. The CCD was cooled down thermoelectrically to $-50{ }^{\circ} \mathrm{C}$. Good pixel events were sorted by setting appropriate energy windows on the CCD. This allowed us to strongly reduce background and higher diffraction order events.

The diagram and hypersatellite $\mathrm{x}$-ray spectra were observed at 12 different photon beam energies ranging from 3122 up to $5451 \mathrm{eV}$. To avoid multiple-hit events on the CCD camera, for the measurements of the strong $K \alpha_{1,2}$ diagram transitions an acquisition time of 1 s/image was used and the beam intensity was attenuated by inserting a $10 \mu \mathrm{m}$ thick $\mathrm{Al}$ absorber in the beam, upstream to the target. As the double $1 s$ photoionization cross section is weak and because, in addition, for aluminum the $K \alpha$ hypersatellite transitions lie above the $K$ edge, which results in an increased selfabsorption of the $K \alpha^{h}$ in the target, longer acquisition times of 10 s/image were chosen for the measurements of the hypersatellite spectra. In order to check the reproducibility of the measurements and the stability of the experimental setup, the hypersatellite spectra were observed in several short successive measurements of typically 200 images. Before each new short measurement the beam intensity was determined with a photodiode and the $K \alpha_{1,2}$ diagram lines were measured with the crystal spectrometer. If necessary, the hypersatellite spectra were then corrected offline for any fluctuations of the beam intensity and beam-spot profile.

\section{Data analysis}

The energy calibration of the X-ray spectra was performed by measuring the $K \alpha_{1,2}$ diagram lines of $\mathrm{Al}$ and $\mathrm{Si}$ and by assigning to these measured lines the corresponding transition energies taken from Ref. [22]. The spectra were fitted by means of a least-squares fitting program, employing Voigt functions for all observed transitions. Voigt functions were used because they correspond to the convolution of the Lorentzian natural line shapes of the transitions with the Gaussian instrumental response of the spectrometer. The energy differences $E\left(K \alpha_{1}\right)-E\left(K \alpha_{2}\right)$ and the Lorentzian widths were kept fixed at the values quoted in Refs. [22,23], respectively. From the fits, Gaussian full widths at half maximum (FWHMs) of 0.75 and $0.78 \mathrm{eV}$ were found for the $K \alpha$ lines of $\mathrm{Al}$ and $\mathrm{Si}$, respectively. The instrumental broadening cor- 


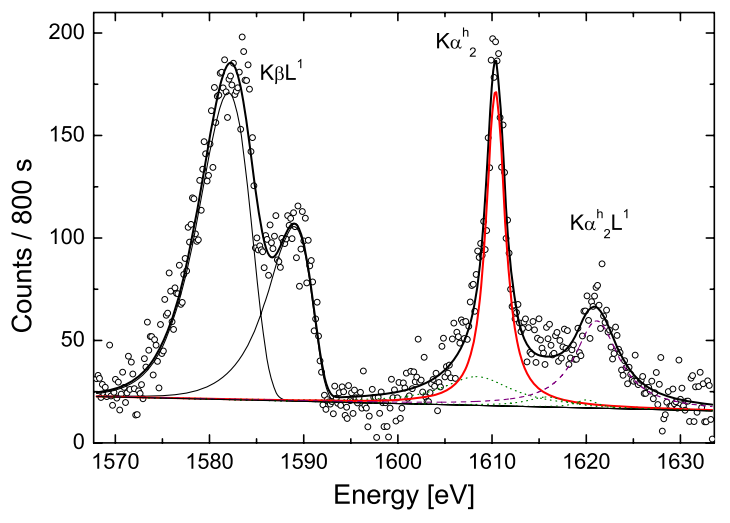

FIG. 1. (Color online) High-resolution $K \beta L^{(1,2)}+K \alpha^{h}$ x-ray spectrum of $\mathrm{Al}$ recorded at an incident photon beam energy of 4786 $\mathrm{eV}$. Open circles correspond to the measurement, the black thin solid lines at about 1580 and $1590 \mathrm{eV}$ to the $K \beta L^{(1)}$ satellite, the dotted green lines to the $K \beta L^{(2)}$ satellite, the red thick solid line at about $1610 \mathrm{eV}$ to the $K \alpha^{h}$ hypersatellite, and the dashed purple line at about $1620 \mathrm{eV}$ to the $K \alpha^{h} L^{(1)}$ hypersatellite satellite.

responding to the $\mathrm{Al} K \alpha$ hypersatellite was determined by interpolation using the fitted Gaussian widths of the $\mathrm{Al}$ and Si $K \alpha_{1,2}$ diagram lines.

High resolution was crucial for these measurements since the $\mathrm{Al} K \alpha$ hypersatellite is very close in energy to the $K \beta L^{(1)}$ satellite and completely overlapping with the $K \beta L^{(2)}$ satellite. Figure 1 shows the $K \beta L^{(1,2)}+K \alpha^{h}$ x-ray spectrum measured at a beam energy of $4786 \mathrm{eV}$. The first doublet corresponds to the components of the $K \beta L^{(1)}$ satellite structure, the third peak at about $1610 \mathrm{eV}$ to the overlapping $K \alpha^{h}$ hypersatellite and $K \beta L^{(2)}$ satellite, while the fourth one corresponds to the $K \alpha^{h} L^{(1)}$ transition, i.e., to the first-order $L$ satellite of the $K \alpha$ hypersatellite. In order to study the dependence on the beam energy of the $\mathrm{Al} K \alpha$ hypersatellite intensity, the shape and relative intensity of the overlapping $K \beta L^{(2)}$ satellite first had to be determined accurately. The measurement of the $K \beta L^{(2)}$ structure was performed at a beam energy of $3122 \mathrm{eV}$ which is smaller than the threshold energy for the double $K$-shell ionization. The obtained spectrum is shown in Fig. 2. The $K \beta L^{(2)}$ spectrum has a complex shape since the $L$ spectator vacancies can be located in different subshells and different couplings are possible between the three holes in the initial and final excited states. KeskiRahkonen et al. [24] reported that four $K \beta L^{(2)}$ satellite transitions are allowed in the $L S$ coupling scheme. As shown in Fig. 2, in our case, only three components were needed to fit the measured spectrum.

The beam energy of $3122 \mathrm{eV}$ was the lowest one used in the present experiment. According to multiconfiguration Dirac-Fock (MCDF) calculations, the threshold energy for producing $K^{-1} L^{-2}$ triple vacancy states in $\mathrm{Al}$ is $1834 \mathrm{eV}$, i.e., significantly lower than the beam energy employed to measure the shape of the pure $K \beta L^{(2)}$ satellite. It can thus be expected that the shape of the $K \beta L^{(2)}$ satellite remains the same at higher beam energies. A similar conclusion was drawn by Fritsch et al. [25] for the $K \alpha_{3,4}$ satellite spectrum of $\mathrm{Cu}$. They found indeed that the shape of the spectrum was varying with the incident photon energy within an energy

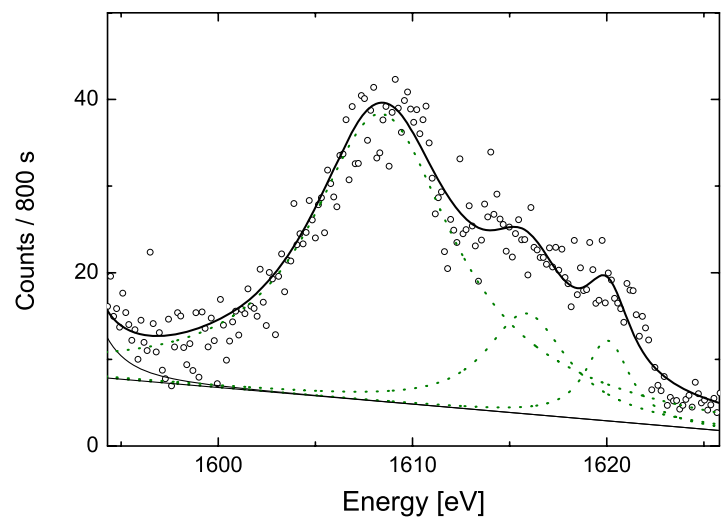

FIG. 2. (Color online) Measured (open circles) and fitted (dotted green lines) $K \beta L^{(2)} \mathrm{x}$-ray spectrum of $\mathrm{Al}$ measured at a photon beam energy of $3122 \mathrm{eV}$.

range of about $50 \mathrm{eV}$ above the threshold and then remained unchanged for higher incident photon energies. They explained the change in the satellite spectrum shape near the threshold region by the variation in the relative intensities of the individual satellite components whose threshold energies are slightly different. As the $K \beta L^{(2)}$ to $K \beta$ intensity ratio should be constant for beam energies far above the threshold and the absolute intensity of the $K \beta$ transition is proportional to the cross section $\sigma_{K}$ for a single $1 s$ ionization, the intensity of the $K \beta L^{(2)}$ satellite measured at higher beam energies was determined by multiplying the satellite intensity measured at $3122 \mathrm{eV}$ by the cross-section ratio $\sigma_{K}\left(E_{\text {beam }}\right) / \sigma_{K}(3122 \mathrm{eV})$.

The fitted $K \alpha^{h}$ spectrum recorded at $4786 \mathrm{eV}$ is shown in Fig. 1. The $K \beta L^{(2)}$ structure was reproduced by three Voigtian functions, whose centers and linewidths were kept fixed at the values obtained from the fit of the spectrum measured at $3122 \mathrm{eV}$. Their intensities were also kept fixed at the values obtained at $3122 \mathrm{eV}$ but multiplied by the intensity ratio $I\left[K \alpha\left(E_{\text {beam }}=4786 \mathrm{eV}\right)\right] / I\left[K \alpha\left(E_{\text {beam }}=3122 \mathrm{eV}\right)\right]$. The latter ratio which is equal to the ratio $\sigma_{K}\left(E_{\text {beam }}\right) / \sigma_{K}(3122 \mathrm{eV})$ was preferably chosen because it presents the advantage to have been determined with the same experimental conditions as the intensity of the $K \beta L^{(2)}$ satellite. To reproduce the $K \alpha^{h}$ hypersatellite and its $L$ satellite, two additional Voigtians were included in the fit shown in Fig. 1. Except the Gaussian widths which were fixed at the values corresponding to the known instrumental resolution of the spectrometer, all other fitting parameters were let free. Due to the asymmetries on their low-energy sides, the two components of the $K \beta L^{(1)}$ satellite were found to be better reproduced by using Pearson functions. Actually, since these two transitions do not evince Lorentzian tails on their highenergy side, they should have only a minor influence on the fit of the $K \alpha^{h}$ hypersatellite. To check this assumption, the $K \alpha$ hypersatellite region above $1595 \mathrm{eV}$ was reanalyzed, without considering the $K \beta L^{(1)}$ transitions. The results given by the second fit were found to be fully consistent with those obtained from the fit of the whole spectrum, differences of only 0.003 and $0.11 \mathrm{eV}$ being observed between the two fits for the $K \alpha^{h}$ energy and natural linewidth, respectively. For this reason, for all other beam energies, only the region of the $K \alpha$ hypersatellite was analyzed. 
TABLE I. Raw and corrected $I\left(K \alpha^{h}\right) / I(K \alpha)$ intensity ratios, double to single $1 s$ photoionization crosssection ratios $P_{K K}$, and double $1 s$ photoionization cross sections $\sigma_{K K}$ as a function of the incident photon energy. For the energies of 4380 and $5120 \mathrm{eV}$, the beam intensity monitoring system was not working properly so that the ratios $I\left(K \alpha^{h}\right) / I(K \alpha)$ could not be determined $\left(5.2(2.2)[-6]\right.$ means $\left.(5.2 \pm 2.2) \times 10^{-6}\right)$.

\begin{tabular}{|c|c|c|c|c|}
\hline $\begin{array}{l}\text { Beam energy } \\
(\mathrm{eV})\end{array}$ & {$\left[\frac{I\left(K \alpha^{h}\right)}{I(K \alpha)}\right]_{\mathrm{fit}}$} & {$\left[\frac{I\left(K \alpha^{h}\right)}{I(K \alpha)}\right]_{\text {cor }}$} & $P_{K K}$ & $\begin{array}{c}\sigma_{K K} \\
\text { (barn) }\end{array}$ \\
\hline 3122 & $5.2(2.2)[-6]$ & $8.5(3.8)[-6]$ & $6.9(3.1)[-6]$ & $0.20(9)$ \\
\hline 3186 & $2.44(33)[-5]$ & $4.00(71)[-5]$ & $3.2(1.2)[-5]$ & $0.89(34)$ \\
\hline 3235 & $2.30(31)[-5]$ & $3.79(66)[-5]$ & $3.1(1.1)[-5]$ & $0.81(30)$ \\
\hline 3318 & $3.13(17)[-4]$ & $5.15(64)[-5]$ & $4.16(56)[-4]$ & $10.3(1.5)$ \\
\hline 3400 & $5.04(27)[-4]$ & $8.30(1.03)[-4]$ & $6.71(90)[-4]$ & $15.5(2.2)$ \\
\hline 3507 & $6.63(35)[-4]$ & $1.09(14)[-3]$ & $8.82(1.17)[-4]$ & $18.7(2.7)$ \\
\hline 3742 & $7.72(42)[-4]$ & $1.27(16)[-3]$ & $1.03(14)[-3]$ & $18.2(2.6)$ \\
\hline 3972 & $9.53(49)[-4]$ & $1.58(19)[-3]$ & $1.27(17)[-3]$ & $19.2(2.7)$ \\
\hline 4786 & $1.37(7)[-3]$ & $2.28(28)[-3]$ & $1.84(25)[-3]$ & $16.5(2.4)$ \\
\hline 5451 & $1.35(7)[-3]$ & $2.25(28)[-3]$ & $1.82(25)[-3]$ & 11.1(1.6) \\
\hline
\end{tabular}

The fitted yields of the diagram and hypersatellite lines were first normalized for the beam intensity and acquisition time. The yield ratios $I\left(K \alpha^{h}\right) / I(K \alpha)$ were then corrected to account for the differences of the self-absorption in the target (correction factor $F_{T}$ ), solid angle of the spectrometer $\left(F_{\Omega}\right)$, crystal reflectivity $\left(F_{X}\right)$, and quantum efficiency of the CCD detector $\left(F_{\mathrm{QE}}\right)$,

$$
\left[\frac{I\left(K \alpha^{h}\right)}{I(K \alpha)}\right]_{\mathrm{cor}}=\left[\frac{I\left(K \alpha^{h}\right)}{I(K \alpha)}\right]_{\mathrm{fit}} F_{T} F_{\Omega} F_{X} F_{\mathrm{QE}}
$$

The self-absorption in the target was taken into account by multiplying the fitted $K \alpha$ and $K \alpha^{h}$ intensities with the following correction factors:

$$
\begin{aligned}
& F_{\mathrm{cor}, K \alpha, K \alpha h} \\
& \quad=\frac{h\left[\mu_{\text {beam }} / \cos (\varphi)+\mu_{K \alpha, K \alpha h} / \sin \left(\varphi+\theta_{K \alpha, K \alpha h}\right)\right]}{1-\exp \left\{-h\left[\mu_{\text {beam }} / \cos (\varphi)+\mu_{K \alpha, K \alpha h} / \sin \left(\varphi+\theta_{K \alpha, K \alpha h}\right)\right]\right\}} .
\end{aligned}
$$

In Eq. (2), $\mu_{\text {beam }}$ and $\mu_{K \alpha, K \alpha h}$ represent the absorption coefficients of Al taken from [26] for the incident photons, respectively, for the diagram and hypersatellite fluorescence $\mathrm{x}$ rays, $\theta_{K \alpha, K \alpha h}$ are the Bragg angles of the two transitions, $\varphi$ is the angle between the normal to the target and the beam direction, and $h$ stands for the target thickness. The factor $F_{T}$ occurring in Eq. (1) is defined by $F_{T}=F_{\text {cor, } K \alpha h} / F_{\text {cor, } K \alpha}$. It was found to vary smoothly with the beam energies between $1.488(32)$ at $E_{\text {beam }}=3122 \mathrm{eV}$ and $1.507(32)$ at $E_{\text {beam }}$ $=5451 \mathrm{eV}$. For the solid angle, crystal reflectivity, and quantum efficiency of the CCD detector, correction factors $F_{\Omega}$ $=1.046(59), F_{X}=1.021(82)$, and $F_{\mathrm{QE}}=1.033(52)$ were obtained. For the crystal reflectivity and CCD quantum efficiency corrections, needed data were taken from Refs. $[27,28]$, respectively. The raw and corrected hypersatellite to diagram yield ratios are given in Table I.

\section{B. Measurements with electrons}

\section{Experimental setup}

The measurements of the Al $K \alpha$ hypersatellite induced by electron impact were performed at the University of Fribourg using a thermoionic electron gun equipped with a Ta disk cathode and electrostatic focusing and deflection electrodes. With the Ta cathode, the electron gun can be operated safely at a pressure of about $10^{-6}$ mbar which corresponds quite well to the vacuum in the spectrometer chamber for standard operation. Accelerating voltage can be varied from $50 \mathrm{~V}$ to $20 \mathrm{kV}$ with a precision of $1 \mathrm{~V}$. The beam current is independently adjustable from $1 \mu \mathrm{A}$ to $1 \mathrm{~mA}$. The power supply of the electron gun offers a feedback stabilized emission current control (ECC) option to maintain a beam current stability of $0.1 \% / \mathrm{h}$.

The setup of the von Hamos spectrometer was very similar to the one used for the synchrotron-radiation measurements. The electron gun was mounted on the same beam port of the spectrometer chamber as the one used at the ESRF. For all measurements, the Al target surface was perpendicular to the electron beam. The energy calibration of the spectrometer and instrumental broadening of the latter were again determined from measurements of the $K \alpha_{1,2}$ lines of $\mathrm{Al}$ and $\mathrm{Si}$. To reduce the background, a cylindrical collimator was installed between the electron gun and the target and the spectrometer slit was covered with a $22 \mu \mathrm{m}$ thick Be foil. To avoid the accumulation of charges in the target, collimator, and surrounding shielding, the latter were carefully grounded.

Due to the electron bombardment, large amounts of heat were produced in the target. For an $\mathrm{Al}$ foil of $1 \mu \mathrm{m}$ it was found that after a few minutes of irradiation, using a beam current of a few hundreds of microamperes, the target part hit by the beam started to melt. Furthermore, as the maximum range in $\mathrm{Al}$ of $8 \mathrm{keV}$ electrons is about $1 \mu \mathrm{m}$, for electron-beam energies higher than $8 \mathrm{keV}$ enhanced target $\mathrm{x}$-ray fluorescence yields are obtained by using thicker tar- 
gets. For these reasons we have opted for a thicker $\mathrm{Al}$ foil of $10 \mu \mathrm{m}$. The latter was found to resist quite well to the heat load even in the case of long term electron irradiation. On the other hand, as already reported in [29], chemical reactions were observed in the target as a result of the electron irradiation. After a few hours of operation, a black dot coinciding in position and shape with the beam spot usually appeared on the surface of the sample. An electron microprobe analysis of this black dot showed that the latter consisted of carbon and oxygen originating from chemical reactions between the heated $\mathrm{Al}$ foil and some residual oil vapor in the spectrometer chamber. It was found that the intensity of the target $\mathrm{x}$-ray emission decreases with time due to this burning effect. For that reason, a short measurement of the $K \beta$ diagram line was performed periodically, and a new $\mathrm{Al}$ foil was installed whenever a change in the $K \beta$ intensity was detected.

The Al spectra were measured with 13 different electronbeam energies ranging between 4 and $20 \mathrm{keV}$. As for the synchrotron-radiation measurements, the hypersatellite lines were measured with a CCD exposure time of $10 \mathrm{~s}$, while the $K \alpha$ diagram lines were measured with an exposure time of 1 s. For each beam energy, the hypersatellite data were collected in several successive measurements of typically 500 images. Before each new measurement, a short measurement of the parent $K \beta$ line was performed to check the stability of the electron-beam intensity and reproducibility of the experimental setup. Depending on the electron energy, beam currents ranging between 140 and $600 \mu \mathrm{A}$ were used. Furthermore, as the shape of the beam profile on the target was found to vary somewhat with the beam current, for each beam energy the $K \alpha_{1,2}$ spectrum was measured with the same current as the one used for the corresponding $K \alpha^{h}$ spectrum. However, to avoid multiple-hit events on the CCD, an absorber $(125 \mu \mathrm{m} \mathrm{Be}+10 \mu \mathrm{m} \mathrm{Al})$ was inserted between the slit and the crystal to decrease the $K \alpha_{1,2}$ line intensity. As this number was crucial for a correct determination of the hypersatellite to diagram line yield ratios, the effective attenuation factor was determined experimentally. A value of $222 \pm 8$ was found.

\section{Data analysis}

As in the present experiment thick targets $\left(\sim 2.7 \mathrm{mg} / \mathrm{cm}^{2}\right)$ were used, the observed $\mathrm{x}$-ray yields should be related to the average energy of the electrons in the target which is given by the following formula [29]:

$$
\bar{E}_{K, K K}=\frac{\int_{0}^{h_{K, K K}} E(x) \sigma_{K, K K}[E(x)] \exp \left[-\frac{\mu_{K \alpha, K \alpha h} x}{\sin \left(\theta_{K \alpha, K \alpha h}\right)}\right] d x}{\int_{0}^{h_{K, K K}} \sigma_{K, K K}[E(x)] \exp \left[-\frac{\mu_{K \alpha, K \alpha h} x}{\sin \left(\theta_{K \alpha, K \alpha h}\right)}\right] d x},
$$

where $\bar{E}_{K}$ and $\bar{E}_{K K}$ represent the average energies of the electrons that produce in the target single and double $K$ vacancies, respectively, $\sigma_{K}[E(x)]$ and $\sigma_{K K}[E(x)]$ are the corresponding cross sections for the electron energy $E(x), \mu_{K \alpha, K \alpha h}$ and $\theta_{K \alpha, K \alpha h}$ are the mass attenuation coefficients and Bragg

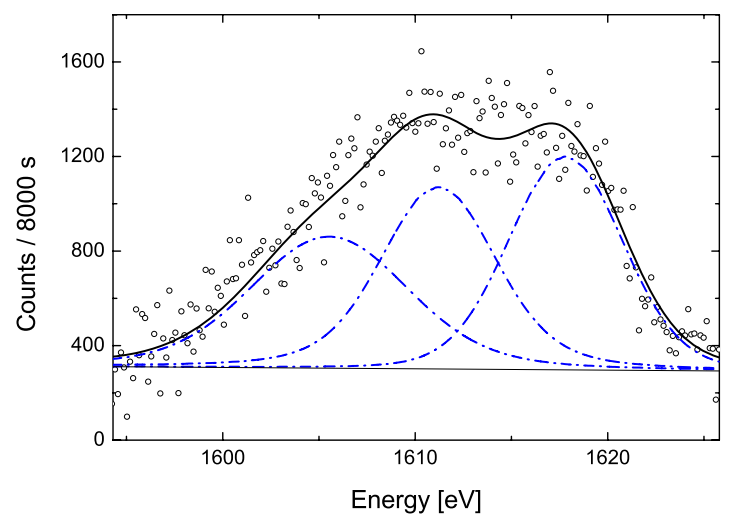

FIG. 3. (Color online) Al oxide x-ray spectrum observed with 4 $\mathrm{keV}$ electrons. Open circles correspond to the measurement; the dashed-dotted blue lines to the fitted transitions.

angles for the diagram and hypersatellite transitions, and $x$ is the projectile penetration depth projected on the axis perpendicular to the target surface. The mass attenuation coefficients were taken from values reported in Ref. [25] and the electron energy was determined from the electron stopping power given by the following expression:

$$
\frac{d E}{d x}=-c E^{-\nu}(x) \Rightarrow E^{\nu}(x) d E=-c d x .
$$

The coefficients $c$ and $\nu$ were derived from the stoppingpower values reported in Ref. [30] and the energy $E(x)$ of the electrons at the depth $x$ was obtained by integrating Eq. (4),

$$
E(x)=\left[E_{e}^{\nu+1}-(\nu+1) c x\right]^{1 /(\nu+1)},
$$

where $E_{e}$ stands for the energy of the incoming electrons. The distances $h_{K}$ and $h_{K K}$ in Eq. (3) represent the penetration depths at which the electron energy is equal to the threshold energy $E_{\mathrm{thr}, K}$ for a single $1 s$ ionization and the threshold energy $E_{\mathrm{thr}, K K}$ for a double $1 s$ ionization, respectively. These distances can be deduced from Eq. (5),

$$
h_{K, K K}=\frac{E_{e}^{\nu+1}-E_{\mathrm{thr}, K, K K}^{\nu+1}}{(\nu+1) c} .
$$

A thin oxide layer is formed on the surface of pure aluminum foils when the latter are exposed to air. Depending on the surface treatment, the oxide layer thickness ranges from a few nanometers to a few hundreds of nanometers. For the lowest beam energy used in this experiment $\left(E_{e}=4 \mathrm{keV}\right)$, a penetration depth $h_{K K}=0.08 \mu \mathrm{m}$ is found from Eq. (6). Therefore, the hypersatellite x-ray spectrum measured at 4 $\mathrm{keV}$ (see Fig. 3) corresponds mainly to transitions in the aluminum oxide layer. The spectrum was fitted with three Voigt functions with all parameters let free in the fit. For higher electron-beam energies the penetration depth increases, so that the relative contribution of the oxide layer with respect to the bulk Al diminishes. Figure 4(a) shows the $K \alpha$ hypersatellite spectrum measured at $E_{e}=6 \mathrm{keV}$. The latter was analyzed in the following way. To fit the $\mathrm{Al}$ oxide $K \beta L^{(2)}$ structure three Voigt functions were used. Except for the intensities of these three Voigtians and the linear back- 


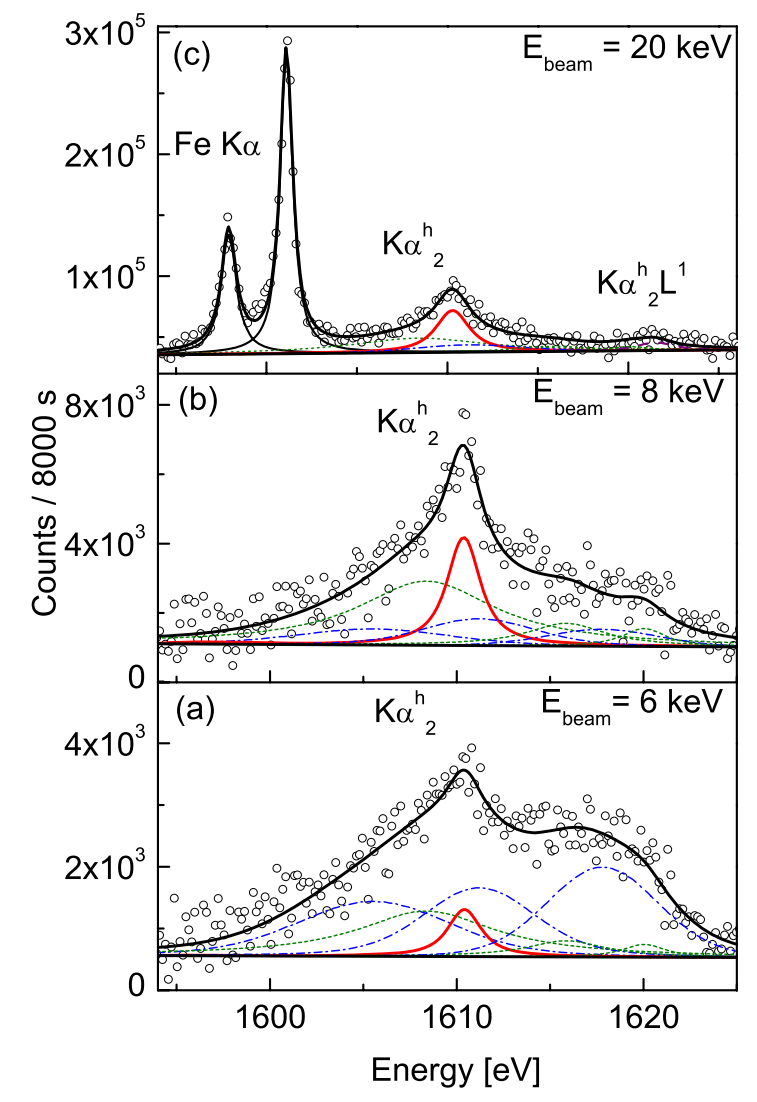

FIG. 4. (Color online) $\mathrm{Al} K \alpha^{h}$ spectra for three different electron-beam energies. The open circles correspond to the experimental points, the solid black lines to the fitted sum spectra, the dashed-dotted blue lines to the $\mathrm{Al}$ oxide transitions, the dotted green lines to the $K \beta L^{(2)}$ satellites, the red lines to the $K \alpha^{h}$ hypersatellites, and the dashed purple line to the $K \alpha^{h} L^{(1)}$ satellite. The two strong lines occurring in the $20 \mathrm{keV}$ spectrum correspond to the $K \alpha_{1,2}$ transitions of $\mathrm{Fe}$ (for details, see text).

ground, all other fitting parameters were set at the same values as the ones obtained from the fit of the Al oxide spectrum measured at $E_{e}=4 \mathrm{keV}$, the intensity ratios of the three Voigtians included. The $\mathrm{Al} K \beta L^{(2)}$ structure was also fitted with three Voigt functions. The energies and widths of the latter and the relative intensities of the two weakest components relative to the strongest one at about $1608 \mathrm{eV}$ were kept fixed in the fit at the values found for the photoinduced $K \beta L^{(2)}$ spectrum measured with the $3122 \mathrm{eV}$ photon beam. Finally, one additional Voigtian was used for the $K \alpha^{h}$ hypersatellite. The Gaussian standard deviation of the latter was fixed at the known instrumental response $(0.32 \mathrm{eV})$, whereas all other fitting parameters were let free. The same fitting procedure was adopted to fit the $K \alpha$ hypersatellite spectra recorded at higher electron energies. For $11 \mathrm{keV}$ and above, an additional Voigtian was introduced in the fits for the $K \alpha^{h} L^{(1)}$ satellite. Spectra recorded at 8 and $20 \mathrm{keV}$ are shown in Figs. 4(b) and 4(c), respectively. The two peaks occurring in the $20 \mathrm{keV}$ spectrum at 1598 and $1601 \mathrm{eV}$ correspond to the fourth order reflection of the $K \alpha_{2}(E=6391 \mathrm{eV})$ and $K \alpha_{1}(E=6404 \mathrm{eV})$ x-ray lines of iron. The latter originate from trace impurities of this metal in the Al target.

The fitted diagram and hypersatellite yields were normalized for the electron-beam intensity and data collecting time.

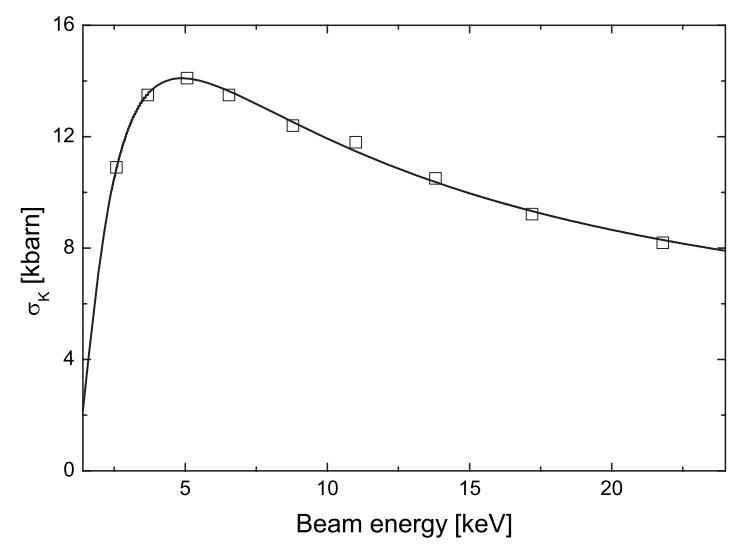

FIG. 5. Electron-induced single $K$-shell ionization cross section $\sigma_{K}$ as a function of the electron energy. The open squares stand for the experimental values reported in [31].

Furthermore, to take into account the effect of the $\mathrm{Be}+\mathrm{Al}$ absorber employed in the measurements of the diagram lines (see Sec. II B 1), the intensities of the latter were multiplied by the measured attenuation coefficient $(222 \pm 8)$. The ratios $I\left(K \alpha^{h}\right) / I(K \alpha)$ were then corrected for the differences in the self-absorption in the target, solid angle of the spectrometer, crystal reflectivity, and quantum efficiency of the CCD, using for the three last correction factors the same values as the ones employed in the photon measurements. The selfabsorption differences were taken into account by multiplying the fitted diagram and hypersatellite intensities with the following correction factors:

$$
F_{\mathrm{cor}, K, K K}=\frac{\int_{0}^{h_{K, K K}} \sigma_{K, K K}[E(x)] d x}{\int_{0}^{h_{K, K K}} \sigma_{K, K K}[E(x)] \exp \left(-\frac{\mu_{K \alpha, K \alpha h} x}{\sin \theta_{K \alpha, K \alpha h}}\right) d x} .
$$

To compute the integrals appearing in formulas (3) and (7), one needs to know the functions $\sigma_{K}(E)$ and $\sigma_{K K}(E)$. The function $\sigma_{K}(E)$ was determined by fitting the experimental cross sections reported in [31] which cover the whole range of electron energies used in our study. The so-determined function $\sigma_{K}(E)$ (see Fig. 5) allowed us to compute the average energies $\bar{E}_{K}$ and the self-absorption factor $F_{\text {cor }, K}$. The obtained values are given in Table II.

The average energies $\bar{E}_{K K}$, correction factors $F_{\text {cor } K K}$, and double $K$-shell ionization cross sections $\sigma_{K K}$ were computed iteratively. The cross sections $\sigma_{K K_{i}}^{(n)}$ corresponding to the $n$th iteration were obtained as follows:

$$
\sigma_{K K_{i}}^{(n)}=\frac{X_{i}\left(K \alpha^{h}\right)}{X_{i}(K \alpha)} \frac{\omega_{K}}{\omega_{K K}} \sigma_{K_{i}}\left(\bar{E}_{K K_{i}}^{(n-1)}\right),
$$

where $X_{i}\left(K \alpha^{h}\right)$ stands for the $K \alpha$ hypersatellite intensity measured at the beam energy $E_{e_{i}}$ and corrected with the selfabsorption coefficient $F_{\mathrm{cor}, K K_{i}}^{(n-1)}$ and $X_{i}(K \alpha)$ stands for the diagram line intensity corresponding to the average energy 
TABLE II. High voltage of the electron gun, average energies $\bar{E}_{K}$ and $\bar{E}_{K K}$ of the electrons producing a single and double $K$-shell ionization, respectively, ratios of the self-absorption correction factors for the measured $K \alpha$ and $K \alpha^{h}$ lines, corrected $K \alpha^{h}$ to $K \alpha$ intensity ratios as well as single and double $K$-shell ionization cross sections. [0.8(3.2)[-6] means $(0.8 \pm 3.2) \times 10^{-6}$.]

\begin{tabular}{ccccccc}
\hline \hline $\begin{array}{c}\mathrm{HV} \\
(\mathrm{kV})\end{array}$ & $\begin{array}{c}\bar{E}_{K} \\
(\mathrm{keV})\end{array}$ & $\begin{array}{c}\bar{E}_{K K} \\
(\mathrm{keV})\end{array}$ & $F_{\mathrm{cor}, K K} / F_{\mathrm{cor}, K}$ & {$\left[X\left(K \alpha^{h}\right) / X(K \alpha)\right]_{\mathrm{cor}}$} & $\begin{array}{c}\sigma_{K}\left(\bar{E}_{K K}\right) \\
(\mathrm{kbarn})\end{array}$ & $\begin{array}{c}\sigma_{K K}\left(\bar{E}_{K K}\right) \\
(\text { barn})\end{array}$ \\
\hline 4 & 3.07 & 3.76 & $1.03(1)$ & $0.8(3.2)[-6]$ & $13.6(1.5)$ & $0.01(4)$ \\
5 & 3.68 & 4.57 & $1.07(1)$ & $4.5(2.1)[-6]$ & $14.1(1.6)$ & $0.05(2)$ \\
6 & 4.29 & 5.38 & $1.12(2)$ & $2.79(61)[-5]$ & $14.0(1.5)$ & $0.32(8)$ \\
7 & 4.89 & 6.18 & $1.19(3)$ & $5.81(1.00)[-5]$ & $13.8(1.5)$ & $0.65(14)$ \\
8 & 5.48 & 6.95 & $1.26(4)$ & $6.50(1.02)[-5]$ & $13.5(1.5)$ & $0.71(14)$ \\
9 & 6.08 & 7.71 & $1.39(5)$ & $8.78(1.31)[-5]$ & $13.1(1.4)$ & $0.93(18)$ \\
10 & 6.67 & 8.48 & $1.53(6)$ & $1.01(15)[-4]$ & $12.7(1.4)$ & $1.04(20)$ \\
11 & 7.27 & 9.29 & $1.70(8)$ & $1.05(16)[-5]$ & $12.3(1.4)$ & $1.04(21)$ \\
13 & 8.47 & 11.01 & $2.07(12)$ & $1.20(18)[-4]$ & $11.5(1.3)$ & $1.12(22)$ \\
15 & 9.69 & 12.86 & $2.59(16)$ & $1.30(19)[-4]$ & $10.7(1.2)$ & $1.13(21)$ \\
17 & 10.40 & 14.80 & $3.16(21)$ & $1.27(31)[-4]$ & $10.0(1.1)$ & $1.03(28)$ \\
18 & 11.57 & 15.79 & $3.55(25)$ & $1.36(22)[-4]$ & $9.7(1.1)$ & $1.07(22)$ \\
20 & 12.87 & 17.79 & $4.35(31)$ & $1.30(10)[-4]$ & $9.2(1.0)$ & $0.97(14)$ \\
\hline \hline
\end{tabular}

$\bar{E}_{K K_{i}}^{(n-1)}$ and corrected with the self-absorption coefficient $F_{\text {cor }, K_{i}}$. The fluorescence yields $\omega_{K}$ and $\omega_{K K}$ of the $1 s^{-1}$ singly ionized and $1 s^{-2}$ doubly ionized $\mathrm{Al}$ atoms were taken from [32]. In the first iteration the approximations $\bar{E}_{K K_{i}}^{(0)} \cong \bar{E}_{K_{i}}$ and $F_{\mathrm{cor}, K K_{i}}^{(0)} \cong F_{\mathrm{cor}, K_{i}}$ were used. The variation in the intensity of the $K \alpha$ line, corrected for the self-absorption, as a function of the average energy $\bar{E}_{K}$ could be well fitted using an exponential function (see Fig. 6). The x-ray yields $X_{i}(K \alpha)$ corresponding to the energies $\bar{E}_{K K_{i}}^{(n-1)}$ were computed using this exponential function.

The values obtained from Eq. (8) for the $\sigma_{K K_{i}}^{(n)}$ were fitted with an appropriate function $\sigma_{K K}^{(n)}(E)$ which permitted us to compute the energies $\bar{E}_{K K_{i}}^{(n)}$ and correction factors $F_{\mathrm{cor}, K K_{i}}^{(n)}$ using Eqs. (3) and (7). The obtained values $\bar{E}_{K K_{i}}^{(n)}$ and $F_{\mathrm{cor}, K K_{i}}^{(n)}$ were then introduced in Eq. (8) to obtain the cross sections

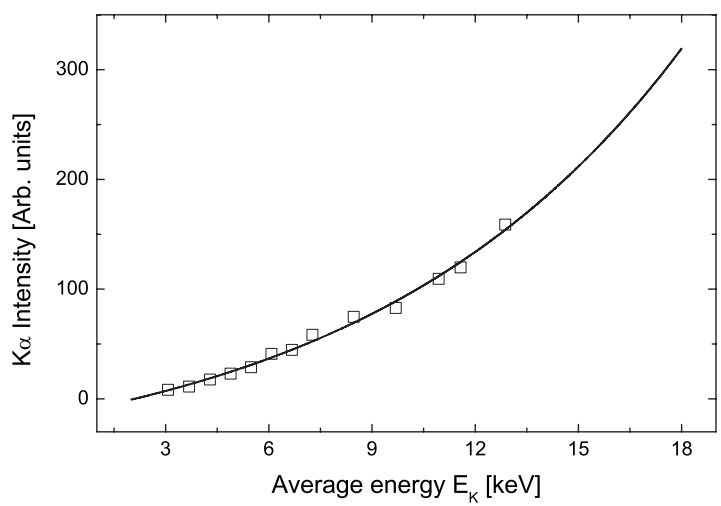

FIG. 6. Variation in the $\mathrm{Al} K \alpha$ intensity corrected for the selfabsorption in the target as a function of the electron average energy. The solid line represents the least-squares fit to the data using an exponential function.
$\sigma_{K K_{i}}^{(n+1)}$, from which the function $\sigma_{K K}^{(n+1)}(E)$ was determined leading to new $\bar{E}_{K K_{i}}^{(n+1)}$ and $F_{\mathrm{cor}, K K_{i}}^{(n+1)}$, and so forth. The method was found to be self-consistent, i.e., after a certain number of iterations (12 in most cases) the average energies $\bar{E}_{K K_{i}}$, the correction factors $F_{\mathrm{cor}, K K_{i}}$, and so the double $K$-shell ionization cross sections $\sigma_{K K_{i}}$ were found to remain unchanged. The final values of the ratios $F_{\mathrm{cor}, K K_{i}} / F_{\mathrm{cor}, K_{i}}$ and cross sections $\sigma_{K K_{i}}$ are listed in Table II. Assuming that the cross sections $\sigma_{K, K K}[E(x)]$ can be replaced in Eq. (7) by their average values $\bar{\sigma}_{K, K K}$, the following expression is found for the correction factors:

$$
F_{\mathrm{cor}, K, K K} \cong \frac{\mu_{K \alpha, K \alpha h} \frac{h_{K, K K}}{\sin \left(\theta_{K, K K}\right)}}{1-\exp \left[-\mu_{K \alpha, K \alpha h} \frac{h_{K, K K}}{\sin \left(\theta_{K, K K}\right)}\right]} .
$$

This simpler expression was used to determine the errors $\Delta\left(F_{\mathrm{cor}, K K} / F_{\mathrm{cor}, K}\right)$ quoted in Table II.

\section{RESULTS AND DISCUSSION}

The obtained results concern only the $K \alpha_{2}^{h}$ hypersatellite $\left({ }^{1} S_{0} \rightarrow{ }^{1} P_{1}\right.$ transition). The $K \alpha_{1}^{h}$ hypersatellite $\left({ }^{1} S_{0} \rightarrow{ }^{3} P_{1}\right.$ transition), which is forbidden by the E1 selection rules in the $L S$ coupling scheme, was indeed too weak to be observed, even for beam energies far above the threshold energy for the double $1 s$ ionization. This is, however, not surprising since, according to theoretical predictions, a value of only $0.87 \%$ is expected for the intensity ratio $I\left(K \alpha_{1}^{h}\right) / I\left(K \alpha_{2}^{h}\right)$ [33].

\section{A. Energy and linewidth of the $K \alpha_{2}^{h}$ hypersatellite}

The energies and linewidths of the $\mathrm{Al} K \alpha_{2}^{h}$ hypersatellite obtained in the present work with the photon and electron 
TABLE III. Energies, linewidths, and relative intensities of the $K \alpha_{2}^{h}$ hypersatellite and $K \alpha_{2}^{h} L^{(1)}$ satellite obtained from the measurements with the photon and electron beams.

\begin{tabular}{lcc}
\hline \hline & Photon impact & Electron impact \\
\hline$K \alpha_{2}^{h}$ energy (eV) & $1610.38(4)$ & $1610.35(7)$ \\
$K \alpha_{2}^{h}$ linewidth (eV) & $1.88(7)$ & $1.87(16)$ \\
$K \alpha_{2}^{h} L^{(1)}$ energy (eV) & $1621.38(14)$ & $1621.93(40)$ \\
$K \alpha_{2}^{h} L^{(1)}$ linewidth $(\mathrm{eV})$ & $2.91(50)$ & a \\
$I\left(K \alpha_{2}^{h} L^{(1)}\right) / I\left(K \alpha^{h}\right)$ & $0.28(1)$ & $0.20(4)$ \\
\hline \hline
\end{tabular}

${ }^{\mathrm{a}}$ It was kept fix in the fit at the value of $2.91 \mathrm{eV}$.

beams are presented in Table III. No dependence on the energy of the synchrotron radiation and electron beam was observed for the energy of the hypersatellite transition nor for its linewidth. Results quoted in Table III correspond therefore to average values of the energies and linewidths observed at different beam energies. However, since for photon beam energies just above the threshold the yields of the hypersatellites were so small that the energies and linewidths of the latter had to be kept fixed in the analysis to be able to fit their intensities, the values corresponding to the lowest photon beam energies were not considered in the calculation of the average energy and linewidth. The same holds for the electron-induced spectra taken at energies $E_{e} \leq 7 \mathrm{keV}$ for which fitting errors were particularly big due to the strong contribution from the $\mathrm{Al}$ oxide layer.

\section{Energy}

From the photon measurements, an average $K \alpha_{2}^{h}$ energy of $1610.38 \mathrm{eV}$ was found. Typical errors provided by the fits for the hypersatellite energy were $\pm 0.02 \mathrm{eV}$. However, if one considers the uncertainty related to the energy calibration of the spectrometer, a total error of about $0.04 \mathrm{eV}$ is obtained. The standard deviation of the nine values taken into consideration for the calculation of the average energy is similar, namely, $0.05 \mathrm{eV}$. From the electron measurements an average $K \alpha_{2}^{h}$ energy of $1610.35 \pm 0.07 \mathrm{eV}$ was obtained which is in excellent agreement with the value found with the photons. For the electron measurements the fit errors were significantly bigger $(0.15-0.25 \mathrm{eV})$ due to the smaller intensity of the hypersatellites induced by electron impact.

The single experimental value reported in the literature is the one published by Keski-Rahkonen et al. [24]. In this study performed with electron beams, an energy shift of the $K \alpha_{2}^{h}$ hypersatellite relative to the parent diagram line of $124.7 \mathrm{eV}$ was found. Using the $K \alpha_{2}$ transition energy of Deslattes et al. [22], this shift gives for the $K \alpha_{2}^{h}$ transition an energy of 1610.99(20), somewhat higher than our value. Although small, the discrepancy might be significant since the difference between the two experimental values $(0.61 \mathrm{eV})$ is bigger than the combined error $(0.21 \mathrm{eV})$. In their recent calculations, Costa et al. [33] computed an energy shift of $124.32 \mathrm{eV}$ relative to the $K \alpha_{1,2}$ diagram lines, which corresponds, using once more the tabulated energies of Deslattes et al. [22], to a value of $1610.82 \mathrm{eV}$ for the $K \alpha_{2}^{h}$ energy. This value is $0.44 \mathrm{eV}$ higher than our result but consistent with it within the combined error if one assumes an uncertainty of $0.6 \mathrm{eV}$ ( $5 \%$ of the calculated energy shift) for the theoretical value. Other available but older and less precise theoretical predictions are $1608 \mathrm{eV}$ [34] and $1627 \mathrm{eV}$ [35].

\section{Linewidth}

For the linewidth of the photoinduced $K \alpha_{2}^{h}$ hypersatellite transition, an average value of $1.88 \mathrm{eV}$ was obtained. Individual fit errors were typically $0.08 \mathrm{eV}$. Including the error of the instrumental broadening of the spectrometer $(\sim 0.06 \mathrm{eV})$, a total error of about $0.07 \mathrm{eV}$ was obtained for the average value. From the fits of the electron-induced spectra an average linewidth of $1.87 \pm 0.16 \mathrm{eV}$ was found. This result is again fully consistent with the value obtained in the photon measurements. Due to the poorer intensity of the electron-induced hypersatellites, individual fit errors were bigger $(0.4-0.6 \mathrm{eV})$ so that the error on the average linewidth is about two times larger than the one corresponding to the photon measurements. The standard deviations of the values taken into account for the calculation of the average linewidths were found to be about $0.1 \mathrm{eV}$ for both types of projectiles.

No result is reported in the literature for the $\mathrm{Al} K \alpha_{2}^{h}$ linewidth. We have thus compared our value with the approximation $3 \Gamma_{K}+\Gamma_{L}$ suggested by Mossé et al. [36]. Using the values recommended by Campbell and Papp [23] for the level widths $\Gamma_{K}$ and $\Gamma_{L}$ of Al, approximation of Mossé et al. [36] gives a width of $1.15 \mathrm{eV}$, a value significantly smaller than our experimental result. Similar findings, however, have been already reported by other groups for $\mathrm{Si}$ [37], $\mathrm{Na}$ [38], and $\mathrm{Cu}$ [14]. Using MCDF calculations, Chen [32] showed that in light atoms $(Z<25)$, the $K$-shell fluorescence yields for double $K$ hole states are higher than those for single $K$ hole states. Therefore, as it was pointed out by Diamant et al. [14], the linewidth of the $K \alpha^{h}$ transition is rather given by $\Gamma_{K K}+\left(\Gamma_{K}+\Gamma_{L}\right)$, where $\Gamma_{K K}$ denotes the width of the initial $1 s^{-2}$ vacancy state. Using the recommended $\Gamma_{K}$ and $\Gamma_{L}$ level widths of Campbell and Papp [23] and the $\Gamma_{K K}$ value extracted from the MCDF calculations of Chen [32], we obtain for the linewidth of the $\mathrm{Al} K \alpha_{2}^{h}$ transition a value of $1.55 \mathrm{eV}$ which is in better agreement with our experimental result, although still smaller by about $20 \%$.

\section{B. $K \alpha_{2}^{h} L^{(1)}$ satellite}

The hypersatellite spectra measured at photon beam energies of 3186, 3318, and $3972 \mathrm{eV}$ are plotted in Fig. 7. In the $3972 \mathrm{eV}$ spectrum, the peak located around $1621 \mathrm{eV}$ corresponds to the $L$ satellite line of the $K \alpha_{2}^{h}$ hypersatellite, i.e., to a transition with three vacancies in the initial and final sates. This peak appears in the photoinduced spectra from a beam energy of $3742 \mathrm{eV}$ and above. The energy given by the fit for the $K \alpha_{2}^{h} L^{(1)}$ satellite is $1621.36(11) \mathrm{eV}$, a value in satisfactory agreement with the result of $1621 \mathrm{eV}$ reported by KeskiRahkonen et al. in [24]. For the width of this transition, an average value of $2.91(50) \mathrm{eV}$ was found. The weak $K \alpha_{2}^{h} L^{(1)}$ transition was also observed in the electron-induced spectra (see Fig. 4) but only for the highest beam energies because of the stronger background characterizing the electron measure- 


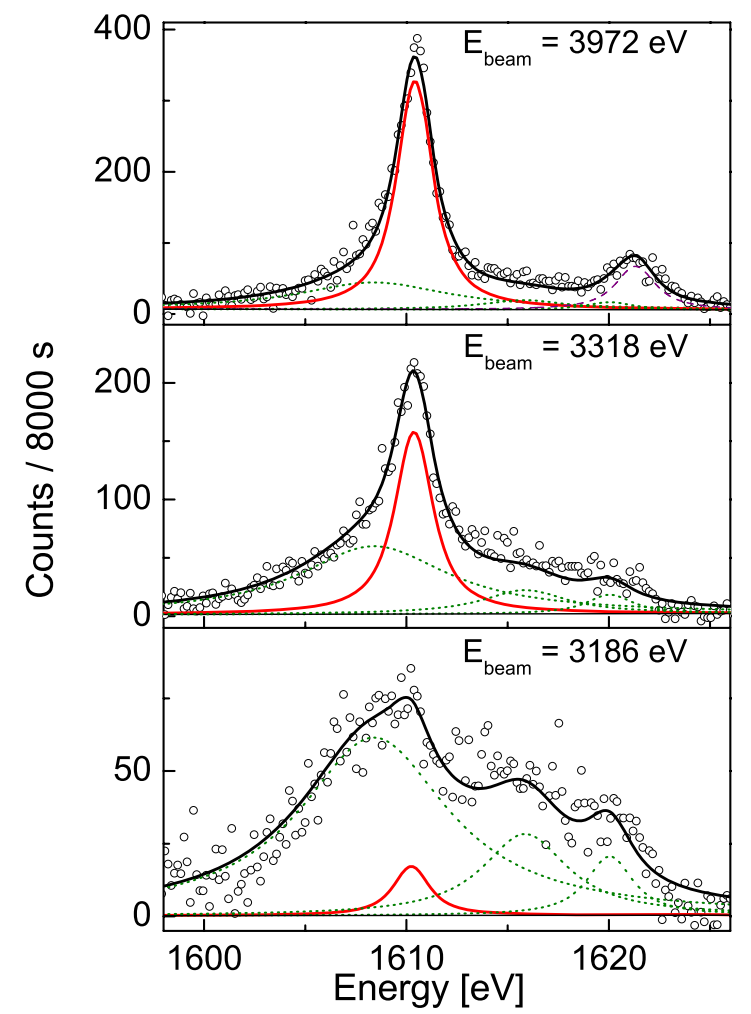

FIG. 7. (Color online) Photoinduced $K \alpha^{h}$ hypersatellite spectra of $\mathrm{Al}$ measured at the indicated beam energies. The dotted green lines correspond to the $K \beta L^{(2)}$ satellites, the red lines to the $K \alpha_{2}^{h}$ hypersatellites, and the dashed purple line to the $K \alpha_{2}^{h} L^{(1)}$ hypersatellite satellite.

ments. An average energy of $1621.93(40) \mathrm{eV}$ was obtained from the electron measurements, whereas the width of the transition could not be determined because it had to be kept fix in the fits at the value obtained from the photon measurements to get reliable intensities.

In first approximation, one could expect the intensity ratios $I\left(K \alpha L^{(1)}\right) / I(K \alpha)$ and $I\left(K \alpha_{2}^{h} L^{(1)}\right) / I\left(K \alpha_{2}^{h}\right)$ to be the same. This assumption, however, is not confirmed by our measurements since, as shown in Table III, values of 0.28(1) (photons) and $0.20(4)$ (electrons) were obtained for the ratio $I\left(K \alpha_{2}^{h} L^{(1)}\right) / I\left(K \alpha_{2}^{h}\right)$, which are three to four times bigger than the value of $0.074(1)$ reported by Mauron and Dousse [29] for the ratio $I\left(K \alpha L^{(1)}\right) / I(K \alpha)$. The same observation was done by Keski-Rahkonen et al. [24] who have reported ratios $I\left(K \alpha_{2}^{h} L^{(1)}\right) / I\left(K \alpha_{2}^{h}\right)=0.42(10) \quad$ and $I\left(K \alpha L^{(1)}\right) / I(K \alpha)$ $=0.098(5)$. This somewhat surprising result, however, can be explained by theory. In a paper concerning measurements of the Auger hypersatellites of $\mathrm{Ne}$ [39], the authors report indeed that, according to shake-off calculations by Foese Fisher [40], the probabilities for observing $L$ satellites in the diagram and hypersatellite $K$ Auger spectra of neon are $16.7 \%$ and $46 \%$, respectively.

\section{Double to single $K$-shell photoionization cross-section ratios}

The ratios of double to single $K$-shell photoionization cross sections $P_{K K}$ and the double $1 s$ photoionization cross

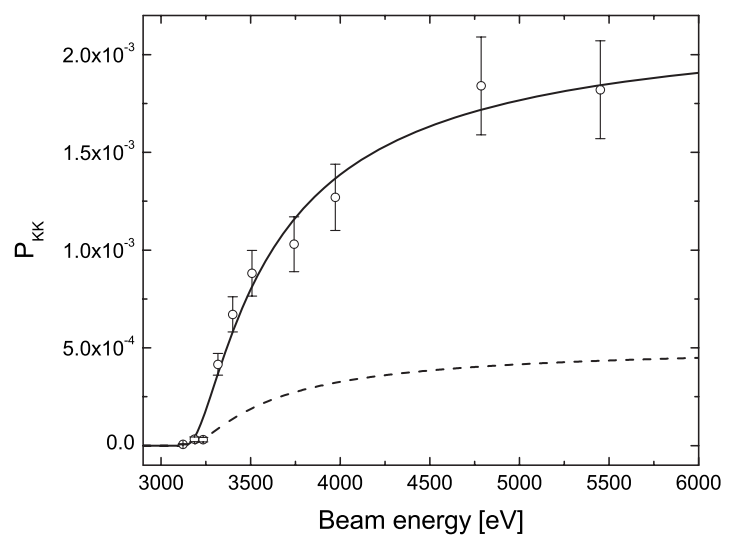

FIG. 8. Ratio of double to single $K$-shell photoionization cross sections $P_{K K}$ as a function of the photon beam energy. The solid line represents the fit to the present experimental data using the Thomas model [44]. The dashed line stands for predictions from the Thomas model using for $P_{K K}(\infty)$ the value corresponding to the asymptotic limit of Forrey et al. [42] (see text for explanations).

sections $\sigma_{K K}$ were determined using the following relations:

$$
\begin{gathered}
P_{K K}=\left[\frac{I\left(K \alpha^{h}\right)}{I(K \alpha)}\right]_{\mathrm{cor}} \frac{\omega_{K}}{\omega_{K K}}, \\
\sigma_{K K}=P_{K K} \sigma_{K},
\end{gathered}
$$

where the corrected intensity ratios $I\left(K \alpha^{h}\right) / I(K \alpha)$ were computed according to Eq. (1), the fluorescence yields $\omega_{K}$ and $\omega_{K K}$ were taken from Ref. [32], and the single-ionization cross sections $\sigma_{K}$ were deduced from the NIST tables [26]. The obtained $P_{K K}$ and $\sigma_{K K}$ values are listed in Table I. Relative uncertainties of $5 \%$ were assumed for the fluorescence yield ratio $\omega_{K} / \omega_{K K}$ and single-ionization cross sections $\sigma_{K}$.

The $P_{K K}$ values are also plotted in Fig. 8 as a function of the photon beam energy. As mentioned before, for the three measurements performed at 3122,3186 , and $3235 \mathrm{eV}$, the $K \alpha^{h}$ structure could be hardly seen in the experimental spectra, whereas for the next-higher beam energy $(3318 \mathrm{eV})$ the hypersatellite clearly emerges from the background. Attempts to fit the $K \alpha^{h}$ hypersatellite observed at $E_{\text {beam }}$ $\leq 3235 \mathrm{eV}$ by keeping fixed the energy and linewidth at the average values obtained from the fits of the spectra taken at higher energies resulted in fitted intensities that are consistent with zero within an uncertainty interval of two to three $\sigma$. It is thus difficult to determine accurately the threshold energy for the double $1 s$ photoionization from our measurements. Nevertheless, considering the straight line obtained from the linear fit of the experimental points corresponding to the beam energies of 3235,3400 , and $3507 \mathrm{eV}$, one finds that the fitted straight line intercepts the $P_{K K}=0$ axis at an energy of $3229 \pm 3 \mathrm{eV}$, a value which is $65 \mathrm{eV}$ lower than the threshold energy predicted by MCDF calculations (3294 $\mathrm{eV})$.

It is well known that due to the combined influence of the knock-out and shake-off processes, the ratio of the double to single photoionization cross sections increases rapidly with excitation energy, levels off at a maximum value (broad 
maximum region), and then drops slowly with growing energy to reach an asymptotic value which corresponds to the photoabsorption shake probability. According to KornbergMiraglia scaling law [41], the region of the broad maximum is reached at an excitation energy given approximately by $0.01 \times Z^{2}(\mathrm{keV})$ above the threshold energy. Assuming for the threshold energy the above mentioned value of $3229 \mathrm{eV}$, this corresponds for Al to a photon beam energy of $4919 \mathrm{eV}$. This estimation is well confirmed by our measurements since, as shown in Fig. 8, the maximum value of the experimental ratio $P_{K K}$ is observed between 4786 and $5451 \mathrm{eV}$.

Recently, Kanter et al. [18] assembled existing experimental ratios of double to single $K$-shell ionization cross sections of elements in the range $2 \leq Z \leq 47$ measured with photon impact and nuclear electron capture in both the broad maximum and asymptotic regions. They found that the $Z$ dependence of the asymptotic ratio scales as $Z^{-2}$ as suggested earlier by Forrey et al. [42] for He-like ions, while the broad maximum ratio scales as $Z^{-1.61}$. Extracting the values corresponding to Al from Fig. 10 in Ref. [42], we obtain an asymptotic ratio of $5.2 \times 10^{-4}$ and a broad maximum ratio of $1.85 \times 10^{-3}$. It can be noted that the latter value agrees very well with our results of $(1.84 \pm 0.25) \times 10^{-3}$ and $(1.82 \pm 0.25) \times 10^{-3}$ obtained at 4786 and $5451 \mathrm{eV}$, respectively. The excitation energy required to reach the photoabsorption asymptotic limit is not yet well known. Previous measurements in $\mathrm{He}$ showed that the asymptotic limit is reached at photon energy of about $3 \mathrm{keV}$ [43], which is $\sim 40$ times bigger than the threshold energy for a double ionization of He. No similar photoabsorption measurements in the asymptotic region are available for higher $Z$ elements but the shake probability of the second $K$ electron can be directly measured for isotopes produced via the nuclear electron capture decay since in this case one of the $1 s$ electrons is absorbed in the nucleus and the second one is released through a shake process [18].

The Thomas model has been used extensively in previous studies to interpret the double photoionization probability. Based on a time-dependent perturbation theory, the energy dependence of the ratio of the double to single $K$-shell photoionization cross sections is described in this model by the following simple formula:

$$
P_{K K}(E)=P_{K K}(\infty) \exp \left(-\frac{r^{2} E_{s}^{2}}{15.32\left(E-E_{\mathrm{thr}}\right)}\right),
$$

where $E_{\mathrm{thr}}$ represents the double $K$-shell ionization threshold energy, $E_{s}$ represents the shake energy, i.e., the energy needed to remove via a shake process the second electron, $P_{K K}(\infty)$ represents the asymptotic shake probability, and $r$ represents a typical distance traveled by the $K$ photoelectron during the atomic potential change. In Eq. (12), the energies are in $\mathrm{eV}$ and the distance $r$ in angstrom. As shown in [45], the distance $r$ can be interpreted as the radius $r_{\max }$ (i.e., the radius at which the radial probability density peaks) of the shaken electron orbital in the ionic atom, i.e., in our case, as the radius $r_{\max }$ of the $1 s$ orbital in the $\mathrm{Al}^{+}$ion. The fit of our data with the Thomas model [44] is shown in Fig. 8. All parameters were let free in the fitting procedure except the shake energy that was fixed to the value of $1669 \mathrm{eV}$ obtained from the difference between the measured threshold energies for the double and single $1 s$ photoionizations. The fit reproduces fairly well the data near the threshold and broad maximum regions and yields a value of $0.047 \AA$ for the $1 s$ radius of $\mathrm{Al}^{+}$, which is in reasonable agreement with the MCDF prediction of $0.040 \AA$. In contrast to that, the fitted threshold energy was found to be $3078 \mathrm{eV}$, which is clearly lower than the above mentioned experimental value of $3229 \mathrm{eV}$ and MCDF prediction of $3294 \mathrm{eV}$. This is, however, not really surprising because similar discrepancies for the threshold energy derived from the Thomas model were also observed by other groups $[13,15]$. Still worse is the fact that the Thomas model gives an asymptotic value $P_{K K}(\infty)$ of $2.21 \times 10^{-3}$ which is more than four times higher than the asymptotic limit of $5.2 \times 10^{-4}$ obtained using the $Z^{-2}$ scaling law of Forrey et al. [42].

Several previous experimental studies have showed that indeed the Thomas model fails to fit the measured results because its simple formalism does not consider the contribution of the KO mechanism which is dominant at lower photon energies. The present work gives therefore further evidence that theoretical models describing more accurately the mechanisms governing the double photoionization probability are needed. Recently, Schneider et al. [46,47] developed a theoretical approach describing the knock-out mechanism as a quasiclassical process and the shake off as a simple quantum process and stated that there is no interference between the two processes. They applied their model to $\mathrm{He}$ and showed that their calculations reproduced quite well the experimental data of Samson et al. [48]. Kanter et al. [18] measured the double to single $K$-shell photoionization crosssection ratio in silver, and rescaling the $\mathrm{SO}$ and $\mathrm{KO} \mathrm{He}$ calculations of Schneider, they were able to fit quite well their data. However, as pointed out by Diamant et al. [15], the scaling of the Schneider calculations performed for He to heavier atoms might be not reliable. Schneider-type calculations for higher $Z$ atoms would thus be welcome to clarify this problem. Assuming that the Thomas model [44] describes only the shake process and locking the asymptotic ratio $P_{K K}(\infty)$ to the value of $5.2 \times 10^{-4}$ obtained from the $Z^{-2}$ scaling law of Forrey et al. [42], we obtain the dashed curve of Fig. 8. The difference between the dashed curve and our experimental data corresponds then to the $\mathrm{KO}$ contribution since according to Schneider et al. [46] there are no interferences between the $\mathrm{KO}$ and $\mathrm{SO}$ processes.

\section{Double to single $K$-shell ionization cross-section ratios for electron impact}

Figure 9 shows the ratios $P_{K K}$ of the double to single $K$-shell ionization cross sections as a function of the average energy $\bar{E}_{K K}$ of the electrons in the sample. As for photons, the broad maximum region could be reached but data clearly beyond the maximum are missing due to the highest possible voltage of $20 \mathrm{kV}$ of the employed electron gun. The ratio reaches a maximum value of $\sim 10^{-4}$ at an average electron energy $\bar{E}_{K K}$ of about $15 \mathrm{keV}$. The single data found in the literature about relative yields of $\mathrm{Al}$ hypersatellites excited 


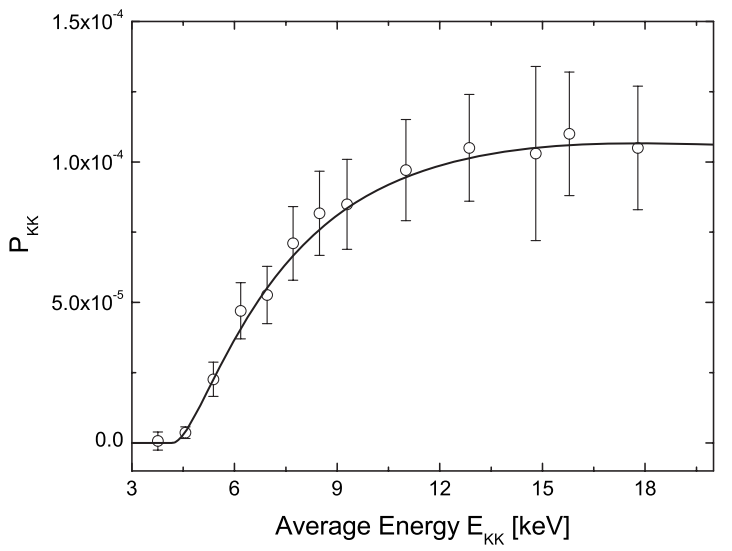

FIG. 9. Ratio of double to single $K$-shell ionization cross sections for electron impact as a function of the average electron energy $\bar{E}_{K K}$. The curve serves only to guide the eye.

by electron impact were reported by Keski-Rahkonen et al. [24]. Using incident electrons of $12 \mathrm{keV}$, they found a value of $1.4(2) \times 10^{-4}$ for the $I\left(K \alpha^{h}\right) / I(K \alpha)$ yield ratio which is consistent within the combined error with the value of 1.13(17) obtained in the present work by interpolating the ratios corresponding to the beam energies of 11 and $13 \mathrm{keV}$ (see Table II).

For the electron-beam energy of $4 \mathrm{keV}$, which corresponds to an average energy $\bar{E}_{K K}$ of $3.76 \mathrm{keV}$, the fitted $K \alpha^{h}$ intensity is consistent with zero within the fit error. For the next-higher energy $\left(\bar{E}_{K K}=4.57 \mathrm{keV}\right)$ a weak but significant hypersatellite yield was provided by the fit so that one can conclude that the threshold energy, in the case of electron impact, should lie between 3.76 and $4.57 \mathrm{keV}$. Considering the intersection of the straight line obtained from the linear fit of the experimental points corresponding to 5,6 , and 7 $\mathrm{keV}$ with the $P_{K K}=0$ axis, a threshold energy of $4.43 \pm 0.08 \mathrm{keV}$ is indeed obtained, which is about $1.2 \mathrm{keV}$ higher than the value obtained from the photon measurements. In our opinion, this apparent discrepancy can be explained by the combined effects of the oxide layer discussed before and the fact that in most collisions, the energy of the incoming electron is shared between the ionized electron and the scattered electron in contrast to photoionization in which the whole photon energy is transferred to the photoelectron.

\section{E. Comparison between the ratios $\boldsymbol{P}_{K K}$ corresponding to photon and electron impact}

In contrast to photons direct multiple ionization is possible for impact with charged particles. A charged particle, like for instance an electron, can indeed interact sequentially with two bound electrons producing a direct double ionization in the target atom. Despite that fact the maximum value of $1.1 \times 10^{-4}$ obtained from the electron measurements for the probability $P_{K K}$ is considerably lower than the corresponding value of $1.8 \times 10^{-3}$ measured with photons. Similar differences were observed in He measurements in which an asymptotic ratio of $1.7 \%$ was found for photoabsorption $[38,49,50]$, whereas for charged particle impact a ratio of only $0.27 \%$ was observed ([51] and references therein). On the other hand, in measurements of the $K \alpha L$ satellites of Al, $\mathrm{Ca}$, and $\mathrm{Co}$ induced by photon and electron impact, Mauron and Dousse [29] found that the cross-section ratios of the photoinduced and electron-induced double $K L$ to single $K$ ionization were very similar. They were even able to estimate the small contribution of the TS2 process by comparing the photoinduced and electron-induced spectra. Likewise, Krause et al. [52] found that the $K \alpha L$ satellites of neon measured with both $3.2 \mathrm{keV}$ electrons and $1.5 \mathrm{keV}$ photons had the same intensity.

To understand this apparent contradiction, one should first notice that the TS2 process is negligibly small for charged projectiles with a ratio $Z / v<0.05[53,54]$, which is the case for the present measurements $(Z$ stands for the projectile charge and $v$ for the projectile velocity expressed in a.u.). Then, it is well known that electron correlations in the same shell are stronger than those between electrons located in different shells. Thus, the fact that the double $K L$ ionization cross sections for photons and electrons are similar, whereas the double $K$ ionization cross sections are different, can be related to electron correlations. Actually, this statement is confirmed by the fact that in the present measurements quite different $K \alpha^{h} / K \alpha$ intensity ratios were observed for photons and electrons, whereas for the $K \alpha^{h} L / K \alpha^{h}$ intensity ratios similar values were obtained.

For charged particle impact, electrons are ejected with a continuous energy distribution and most of them leave the atom with small velocities comparable to their original orbital velocities [53]. Consequently the sudden approximation (SA) is no more valid $[55,56]$. If the outgoing electron leaves the atom with a low velocity, the corresponding atomic orbital has indeed enough time to adapt to the new atomic potential. As a consequence, there will be no overlap between the initial and final wave functions and shake off of a second electron is less likely to occur. Actually, while SO is well defined in the framework of the SA model at the asymptotic energy limit, its meaning when the velocity of the outgoing electron is low is not clear $[57,58]$. We come to the conclusion that for electron impact, the SO process contributes certainly to the observed double ionization but to a significantly smaller extent than in the case of photon impact.

Concerning the KO process, it is well known that its amplitude depends strongly on the velocity of the primary electrons. For photons, at high energies, when the photoelectron velocity exceeds considerably the orbital velocity, the $\mathrm{KO}$ probability vanishes. In contrast to that, in the case of charged particle impact, most electrons continue to be ejected with small velocities, comparable to their original orbital velocities, independently of the projectile energy, so that the $\mathrm{KO}$ probability tends to a constant value at the asymptotic energy limit $[56,59,60]$.

In a series of pioneering works aiming to investigate the relationship between the double ionization induced by photons and charged particles, McGuire and co-workers demonstrated that within the framework of the dipole approximation describing the photoabsorption process (i.e., photoionization ascribed solely to the photoeffect without any contribution from the Compton effect), the ratio of double to single total ionization cross section for He in case 
of photon impact is much higher than that for charged particle impact [59], while being similar to the ratio of double to single differential cross section for charged particle impact corresponding to the same energy of the ejected electron [61]. Above McGuire's statement was confirmed experimentally by Cocke et al. [62] who performed measurements of the double- to single-ionization differential cross section ratio of He bombarded with 1 and 3-MeV protons. By measuring the energy loss of the scattered protons, they were able to isolate the fast ejected electrons and they obtained ratios of about $2 \%$, close to the ratios of double to single total cross section found in the case of photoionization for the same energies of the ejected electrons (600 and $900 \mathrm{eV})$. However, McGuire et al. [53] pointed out that the conclusions in measurements of Cocke et al. [62] should be considered cautiously since at these proton energies a small contribution from the TS2 process is possible. Similar measurements were performed by $\mathrm{Wu}$ et al. [63]. Using 2, 3, and 6-MeV protons, they also found a ratio close to $2 \%$ at an energy transfer of about $1 \mathrm{keV}$. Measurements of DeHaven et al. [64] yield, however, a lower value for this ratio. Using binary kinematics to compute the energy transfer corresponding to their measured proton-scattering angles, they found over a large energy-transfer range, a practically constant ratio of about $1.25 \%$.

As discussed by different authors [53,63,65,66], even when the primary ejected electron leaves the atom after photoabsorption with the same energy as the one ejected after projectile impact ionization, the residual ion does not receive the same recoil momentum in both processes because the photoabsorption and projectile impact ionization processes probe different parts of the wave function in the target atom momentum space. In the case of photoabsorption, momentum conservation requires that the momentum of the recoil ion mirrors the one of the ejected photoelectron, whereas for projectile impact ionization, the momentum lost by the projectile is transferred to the ejected electron and the ion is left with its initial momentum. This situation was suggested to be very similar to ionization induced by Compton scattering. Indeed, measurements in He using the cold-target recoil ion momentum spectroscopy (COLTRIMS) method have confirmed that the momentum distributions of the $\mathrm{He}^{+}$ions are very similar in Compton scattering and projectile impact ionization experiments [67]. On the theoretical side, Burgdörfer et al. [68] demonstrated within the Born approximation that for a given energy transfer to the primary ejected electron the ratio of double to single ionization for projectile impact is equal to the one obtained for Compton scattering.

Direct differential cross-section measurements of $\mathrm{Wu}$ et al. [63] for He using fast protons as projectiles resulted in an asymptotic ratio of $0.8 \%$, which is lower than the value of $1.25 \%$ deduced from the kinematics measurements of DeHaven et al. [64]. No experimental data concerning the differential cross-section ratio for Compton scattering are available in the literature. However, according to Burgdörfer et al. [68], the dominant contribution to the total-cross-section ratio for Compton scattering originates from large energy transfer. Using the COLTRIMS method, Spielberger et al. [69] found that in $\mathrm{He}$ the ratio of total cross section for Compton scattering in the energy range of 40-100 keV ap- pears to be $0.98 \%$, and they noted that over the investigated energy range, the asymptotic ratio was not yet reached. Comparing this value to the well-established $1.7 \%$ asymptotic photoabsorption cross-section ratio, Burgdörfer et al. [68] concluded that the probability for shaking off the second electron differs significantly whether the first electron is removed by photoabsorption or by Compton scattering, in contradiction with a previous prediction of Mukoyama et al. [70], stating that in the sudden approximation, the shake probability is independent of the primary ionization mechanism. Experimental data concerning the asymptotic ratios for Compton scattering and charged particle impact are not available for heavier elements. Our present measurements in Al do not reach the asymptotic region but confirm that the total cross-section ratio in the broad maximum region is clearly smaller for charged particle impact than for photon impact.

\section{CONCLUDING REMARKS}

The energy dependence of the $K \alpha$ hypersatellite x-ray spectrum of $\mathrm{Al}$ was measured using synchrotron radiation and electrons for the production of the double $1 s$ vacancies. The measurements were performed by means of highresolution $\mathrm{X}$-ray spectroscopy, employing a von Hamos crystal spectrometer. The $K \alpha_{2}^{h}$ transition energies and natural linewidths were determined from the measurements performed with both photons and electrons. Consistent values of $1610.38(10)$ and 1610.37(11) eV and 1.88(10) and 1.87(17) $\mathrm{eV}$ were obtained for the hypersatellite energy and linewidth, respectively. Since in light atoms the $K$ shell fluorescence yields for double $1 s$ vacancy states are higher than those of single $1 s$ vacancy states, the $K \alpha_{2}^{h}$ natural linewidth was found to be better reproduced by the formula $\Gamma\left(K \alpha^{h}\right)=\Gamma_{K K}$ $+\left(\Gamma_{K}+\Gamma_{L}\right)$, where $\Gamma_{x}$ stands for the width of the $x$ vacancy state, than by the standard Mossé formula $\Gamma\left(K \alpha^{h}\right)=3 \Gamma_{K}$ $+\Gamma_{L}$. Regarding the $K \alpha_{1}^{h}$ hypersatellite, no trace of this line could be detected in our measurements due to the fact that this transition is strongly hindered by the E1 selection rules within the $L S$ coupling scheme which prevails for light elements like Al.

The energy dependences of the double $K$-shell ionization cross sections were determined from the measured hypersatellite to diagram intensity ratios and the single $K$-shell ionization cross sections reported in the literature. The ratio of the double to single $K$-shell photoionization cross sections was found to reach the broad maximum at about $5 \mathrm{keV}$, in good agreement with the prediction of $4.984 \mathrm{keV}$ derived from the Kornberg's scaling law. For the maximum value of the ratio, a result of $1.84(25) \times 10^{-3}$ was obtained, which is also in good agreement with the value of $1.85 \times 10^{-3}$ deduced from the $1 / Z^{1.61}$ scaling law of Kanter et al. [18]. We have also shown in our study that the Thomas model [44] does not fit properly the evolution of the measured double to single $K$-shell ionization.

A detailed comparison between the data obtained by photoionization and electron impact was also done. Measurements performed by means of electron impact provided a maximum double to single $K$-shell ionization cross-section 
ratio of $1.1 \times 10^{-4}$ considerably smaller than the corresponding ratio of $1.8 \times 10^{-3}$ obtained from the photon measurements. This is, however, not really surprising since similar substantially lower double to single cross-section ratios for charged particle impact ionization compared to photoionization were also found for He by other groups. For He, the differences could be explained theoretically and confirmed by a variety of experiments. The threshold energies for the production of double $1 s$ vacancy states were found to be $3229(3) \mathrm{eV}$ in the case of photoionization and 4432(77) eV for the electron-induced ionization. The difference was explained by the fact that in most electron-electron collisions only a part of the energy of the incident electron is transferred to the photoelectron, whereas in photoionization the whole photon energy is given to the ionized electron.

\section{ACKNOWLEDGMENTS}

The authors would like to thank Dr. D. Reichert and Dr. R. Tucoulou from the ESRF beamline ID21 for providing our experiment with very good beam conditions. We also acknowledge the financial support of the Swiss National Science Foundation and the ESRF.
[1] J. H. McGuire, Electron Correlation Dynamics in Atomic Collisions (Cambridge University Press, Cambridge, England, 1997).

[2] S. L. Carter and H. P. Kelly, Phys. Rev. A 24, 170 (1981).

[3] T. Ishihara, K. Hino, and J. H. McGuire, Phys. Rev. A 44, R6980 (1991).

[4] J. A. R. Samson, Phys. Rev. Lett. 65, 2861 (1990).

[5] R. Dörner et al., Radiat. Phys. Chem. 70, 191 (2004).

[6] J. A. Tanis, R. D. DuBois, and A. S. Schlachter, Phys. Rev. Lett. 68, 897 (1992).

[7] J. P. Briand, P. Chevallier, A. Chetioui, J. P. Rozet, M. Tavernier, and A. Touati, Phys. Rev. A 23, 39 (1981).

[8] T. Åberg et al., J. Phys. B 9, 2815 (1976).

[9] J. P. Briand, P. Chevalier, M. Tavernier, and A. Touati, Phys. Rev. Lett. 27, 777 (1971).

[10] T. A. Carlson, Phys. Rev. 156, 142 (1967).

[11] F W. Byron, Jr. and Ch. J. Joachain, Phys. Rev. 164, 1 (1967).

[12] E. P. Kanter, R. W. Dunford, B. Krassig, and S. H. Southworth, Phys. Rev. Lett. 83, 508 (1999).

[13] M. Oura et al., J. Phys. B 35, 3847 (2002).

[14] R. Diamant, S. Huotari, K. Hamalainen, C. C. Kao, and M. Deutsch, Phys. Rev. A 62, 052519 (2000).

[15] R. Diamant et al., Radiat. Phys. Chem. 75, 1434 (2006).

[16] R. Diamant, S. Huotari, K. Hamalainen, R. Sharon, C. C. Kao, and M. Deutsch, Phys. Rev. Lett. 91, 193001 (2003).

[17] S. H. Southworth, E. P. Kanter, B. Krassig, L. Young, G. B. Armen, J. C. Levin, D. L. Ederer, and M. H. Chen, Phys. Rev. A 67, 062712 (2003).

[18] E. P. Kanter, I. Ahmad, R. W. Dunford, D. S. Gemmell, B. Krassig, S. H. Southworth, and L. Young, Phys. Rev. A 73, 022708 (2006).

[19] C. Dal Cappello, R. El Mkhanter, and P. A. Hervieux, Phys. Rev. A 57, R693 (1998).

[20] A. Dorn, A. Kheifets, C. D. Schroter, C. Hohr, G. Sakhelashvili, R. Moshammer, J. Lower, and J. Ullrich, Phys. Rev. A 68 , 012715 (2003).

[21] J. Hoszowska, J.-Cl. Dousse, J. Kern, and Ch. Rhême, Nucl. Instrum. Methods Phys. Res. A 376, 129 (1996).

[22] R. D. Deslattes et al., Rev. Mod. Phys. 75, 35 (2003).

[23] J. L. Campbell and T. Papp, At. Data Nucl. Data Tables 77, 1 (2001).

[24] O. Keski-Rahkonen, E. Mikkola, K. Reinikainen, and M. Lehkonen, J. Phys. C 18, 2961 (1985).
[25] M. Fritsch, C. C. Kao, K. Hamalainen, O. Gang, E. Forster, and M. Deutsch, Phys. Rev. A 57, 1686 (1998).

[26] http://physics.nist.gov/PhysRefData/Xcom/Text/XCOM.html

[27] B. L. Henke, E. M. Gullikson, and J. C. Davis, At. Data Nucl. Data Tables 54, 181 (1993).

[28] J. Szlachetko et al., Rev. Sci. Instrum. 78, 093102 (2007).

[29] O. Mauron and J.-Cl. Dousse, Phys. Rev. A 66, 042713 (2002).

[30] http://physics.nist.gov/PhysRefData/Star/Text/ESTAR.html

[31] M. Liu et al., At. Data Nucl. Data Tables 76, 213 (2000), and references therein.

[32] M. H. Chen, Phys. Rev. A 44, 239 (1991).

[33] A. M. Costa et al., J. Phys. B 40, 57 (2007).

[34] M. Gavrila and J. M. Hansen, J. Phys. B 11, 1353 (1978).

[35] T. K. Mukherjee and P. K. Mukherjee, Z. Phys. D: At., Mol. Clusters 42, 29 (1997).

[36] J. P. Mossé, P. Chevallier, and J. P. Briand, Z. Phys. A 322, 207 (1985).

[37] M. Kavčič and K. Tökési, Phys. Rev. A 72, 062704 (2005).

[38] J. Auerhammer, H. Genz, G. Kilgus, A. Kumar, and A. Richter, Phys. Rev. A 35, 4505 (1987).

[39] Ž. Šmit, M. Zitnik, L. Avaldi, R. Camilloni, E. Fainelli, A. Muhleisen, and G. Stefani, Phys. Rev. A 49, 1480 (1994).

[40] C. Foese Fisher, The Hartree-Fock Method for Atoms (Wiley, New York, 1977).

[41] M. A. Kornberg and J. E. Miraglia, Phys. Rev. A 49, 5120 (1994).

[42] R. C. Forrey, H. R. Sadeghpour, J. D. Baker, J. D. Morgan, and A. Dalgarno, Phys. Rev. A 51, 2112 (1995).

[43] J. C. Levin, G. B. Armen, and I. A. Sellin, Phys. Rev. Lett. 76, 1220 (1996).

[44] T. D. Thomas, Phys. Rev. Lett. 52, 417 (1984).

[45] P.-A. Raboud, M. Berset, J. C. Dousse, Y. P. Maillard, O. Mauron, J. Hoszowska, M. Polasik, and J. Rzadkiewicz, Phys. Rev. A 65, 062503 (2002).

[46] T. Schneider, P. L. Chocian, and J. M. Rost, Phys. Rev. Lett. 89, 073002 (2002).

[47] T. Schneider and J. M. Rost, Phys. Rev. A 67, 062704 (2003).

[48] J. A. R. Samson, W. C. Stolte, Z. X. He, J. N. Cutler, Y. Lu, and R. J. Bartlett, Phys. Rev. A 57, 1906 (1998).

[49] J. C. Levin, D. W. Lindle, N. Keller, R. D. Miller, Y. Azuma, N. B. Mansour, H. G. Berry, and I. A. Sellin, Phys. Rev. Lett. 67, 968 (1991). 
[50] L. Spielberger et al., Phys. Rev. Lett. 74, 4615 (1995).

[51] J. Ullrich et al., Phys. Rev. Lett. 71, 1697 (1993).

[52] M. O. Krause et al., Phys. Lett. 31, 81 (1970).

[53] J. H. McGuire et al., J. Phys. B 28, 913 (1995).

[54] A. S. Schlachter and J. A. Tanis, Phys. Rev. Lett. 73, 3596 (1994).

[55] S. T. Manson, R. D. DuBois, and L. H. Toburen, Phys. Rev. Lett. 51, 1542 (1983).

[56] L. H. Andersen, P. Hvelplund, H. Knudsen, S. P. Moller, A. H. Sorensen, K. Elsener, K. G. Rensfelt, and E. Uggerhoj, Phys. Rev. A 36, 3612 (1987).

[57] T. Pattard, T. Schneider, and J. M. Rost, J. Phys. B 36, L189 (2003).

[58] T. Pattard and J. Burgdörfer, Phys. Rev. A 64, 042720 (2001).

[59] J. H. McGuire, J. Phys. B 17, L779 (1984).

[60] L. H. Andersen, P. Hvelplund, H. Knudsen, S. P. Moller, K. Elsener, K. G. Rensfelt, and E. Uggerhoj, Phys. Rev. Lett. 57, 2147 (1986).

[61] S. T. Manson and J. H. McGuire, Phys. Rev. A 51, 400 (1995).
[62] C. L. Cocke, R. Dörner, A. D. Gonzalez, and E. HorsdalPedersen, in Abstracts of XVIII International Conference on the Physics of Electronic and Atomic Collisions, edited by T. Andersen, B. Fastrup,F. Folmann,H. Knudsen, and N. A. Andersen (Aarhus University, Aarhus, 1993), p. 845.

[63] W. Wu, S. Datz, N. L. Jones, H. F. Krause, B. Rosner, K. D. Sorge, and C. R. Vane, Phys. Rev. Lett. 76, 4324 (1996).

[64] W. R. DeHaven, C. Dilley, A. Landers, E. Y. Kamber, and C. L. Cocke, Phys. Rev. A 57, 292 (1998).

[65] E. Y. Kamber, C. L. Cocke, S. Cheng, and S. L. Varghese, Phys. Rev. Lett. 60, 2026 (1988).

[66] L. Végh and J. Burgdörfer, Phys. Rev. A 42, 655 (1990).

[67] R. Dörner et al., Phys. Rep. 330, 95 (2000).

[68] J. Burgdörfer, L. R. Andersson, J. H. McGuire, and T. Ishihara, Phys. Rev. A 50, 349 (1994).

[69] L. Spielberger et al., Phys. Rev. A 59, 371 (1999).

[70] T. Mukoyama, Y. Ito, and K. Taniguchi, X-Ray Spectrom. 28, 491 (1999). 